\title{
Evidence of Clumpy Dust Shell Structure in IRC+10216 from K Band Lunar Occultation Observations
}

\author{
T. Chandrasekhar and Soumen Mondal \\ Physical Research Laboratory, Navrangpura, Ahmedabad, India
}

\begin{abstract}
Lunar occultation observations at 2.2 microns of the inner dust shell of the carbon rich long period variable IRC +10216 yield a clumpy structure with atleast four components. Comparison with concurrent and earlier speckle observations confirm the presence of these components and show that real spatial changes are taking place at the level of milli arcseconds on time scales of a few years in the inner dust shell of IRC + 10216 .
\end{abstract}

\section{Introduction}

In recent years near Infrared Speckle interferometry of IRC+10216 has revealed the presence of several compact components within a 200 milli arcsec radius embedded in a fainter, asymmetric nebula (Osterbart et al. 2000). The relative positions and intensities of these compact components are changing over time scales of a few years. The compact components are possibly the result of fragmentation of inner shell structure due to inhomogeneous mass loss process in the rapidly evolving object. In this paper we present the observations and analysis of a recent Lunar occultation of IRC +10216 in the $\mathrm{K}$ band (Table 1).

Table 1. Details of observations

\begin{tabular}{lcl}
\hline Date & $: 1998$ December 08 \\
Event time & $:$ 19:16:36.6 $\pm 0.5 \mathrm{UT}$ \\
Position Angle (NESW) & $:$ 304 deg \\
Lunar Velocity Comp. & $:$ & -0.4156 arcsec/sec \\
Photometric phase & $:$ & 0.67 \\
K Magnitude & $:$ & $2.06 \pm 0.07$ \\
Telescope Location & $:$ & $1.2 \mathrm{~m}$ Gurushikhar, Mt. Abu, India \\
& $:\left(24^{\circ} 39 / 8 / 1.88 \mathrm{~N}, 72^{\circ} 46 / 4711.47 \mathrm{E}, 1680 \mathrm{~m}\right)$ \\
Instrument & $:$ Single element InSb in a LN $\mathrm{LN}_{2}$ cooled dewar \\
& $:$ Chandrasekhar 1999) \\
Filter & $:$ millisecond $(\lambda=2.2 \mu \mathrm{m}, \Delta \lambda=0.4 \mu \mathrm{m})$ \\
Data Sampling &
\end{tabular}



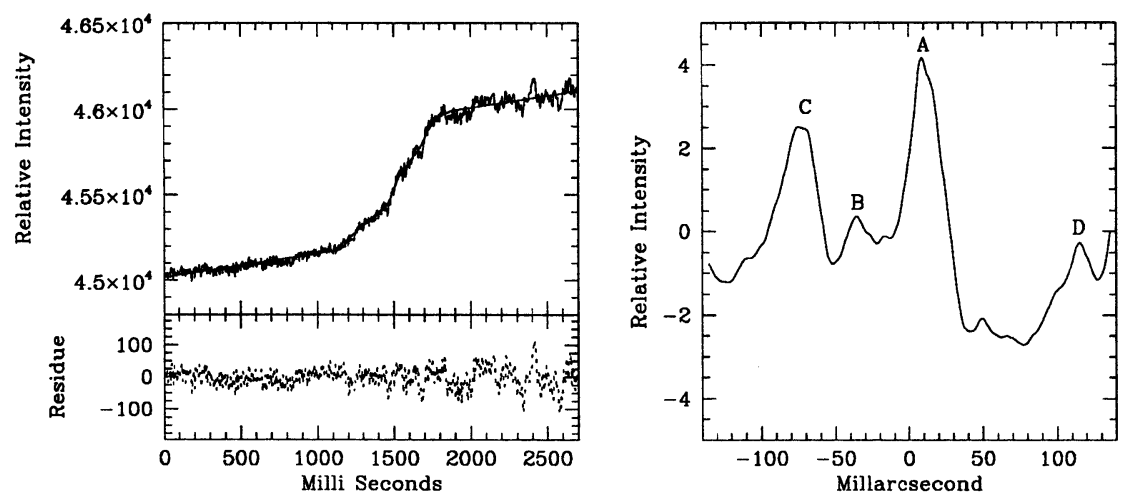

Table 2. Fine Structures in the Dust Shell of IRC+10216

\begin{tabular}{llllll}
\hline $\begin{array}{l}\text { Com- } \\
\text { po- } \\
\text { nent }\end{array}$ & $\begin{array}{l}\text { Location from } \\
\text { Centre of } \\
\text { uniform disk }\end{array}$ & $\begin{array}{l}\text { Projected } \\
\text { Separation } \\
\text { From A }\end{array}$ & $\begin{array}{l}\text { Actual } \\
\text { Separation } \\
\text { From A }\end{array}$ & $\begin{array}{l}\text { Linear } \\
\text { Separation } \\
\left(\mathrm{X} 10^{14} \mathrm{~km}\right) \\
\text { for d=150 pc }\end{array}$ & $\begin{array}{l}\mathrm{R} / \mathrm{R}_{*} \\
\mathrm{R}_{*}= \\
5 \times 10^{13} \mathrm{~cm}\end{array}$ \\
& $(\mathrm{mas})$ & $(\mathrm{mas})$ & $(\mathrm{mas})$ & & \\
\hline $\mathrm{A}$ & +11 & - & - & - & - \\
$\mathrm{B}$ & -34 & 45 & 186 & 4.19 & 8.38 \\
$\mathrm{C}$ & -74 & 85 & 104 & 2.34 & 4.68 \\
$\mathrm{D}$ & +117 & 106 & 206 & 4.64 & 9.28 \\
\hline
\end{tabular}

\section{Analysis, Results and Discussion}

Data analysis involved a model independent algorithm (MIA) incorporating a Non linear least square method with Lucy's deconvolution algorithm. The uniform disk brightness profile of $294 \pm 5$ mas was used as the guess profile in the Lucy algorithm to obtain the final best fit profile which departs significantly from the UD profile signifying a clumpy structure in IRC + 10216 .

Fig. 1 shows the Lunar occultation data and the best fit obtained. Fig. 2 shows the brightness profile (from which UD profile has been substracted) giving the best fit to the data. Atleast four components are seen whose relative positions are given in Table 2, C, B, A and D being the order in which they occur in the direction of occultation. For typical values of distance and stellar radius these separations are listed in linear units. If we assume that components of B, $\mathrm{C}, \mathrm{D}$ have separated from A at the rate of $15 \mathrm{~km} / \mathrm{s}$ then the time scales involved are 5 to 10 years. B and D could well be part of same event while $\mathrm{C}$ might have been ejected earlier.Close monitoring of the inner zone of IRC+10216 at high angular resolution in future will be crucial in revealing the time evolution of the fine structure of the dust shell.

\section{References}

Chandrasekhar, T. 1999, Bull. Astr. Soc. India, 27, 43.

Osterbart, R., Balega, Y. Y., Blocker, T., Menshchikov, A. B., Weigelt, G. 2000, A\&A, 357, 169. 